\title{
Comparative Study of Three Preoperative Imaging Modalities for the Evaluation and Design of Superficial Circumflex Iliac Artery Perforator Flap: Color Doppler Ultrasound, Computed Tomography Angiography and Magnetic Resonance Angiography
}

\author{
Zhuowei Tian ${ }^{\# 1}$, Shizhe Wang ${ }^{\# 1}$, Yue $\mathrm{He}^{1}$ and Chunyue Ma ${ }^{1,{ }^{*}}$ \\ ${ }^{1}$ Shanghai Key Laboratory of Stomatology, Department of Oral and Maxillofacial-Head and Neck Oncology, 9th People's Hospital, Shanghai Jiao Tong University School of \\ Medicine, Shanghai, China \\ "Corresponding author: Shanghai Key Laboratory of Stomatology, Department of Oral and Maxillofacial-Head and Neck Oncology, 9th People's Hospital, Shanghai Jiao Tong \\ University School of Medicine, No. 639, Zhi Zao Ju Road, Shanghai, China. Email: maxifama@163.com \\ \# These authors are contributed equally as the first author.
}

Received 2019 August 17; Revised 2020 June 27; Accepted 2020 July 04.

\begin{abstract}
Background: Superficial circumflex iliac artery perforator (SCIP) flap is a promising reconstructive candidate for head and neck, trunk and extremity reconstruction. In order to reduce intraoperative errors, preoperative planning is essential for evaluation of the possible variations in vascular anatomy of the groin region. However, the use of these modalities has not been compared. Objectives: The three commonly used imaging modalities [color Doppler ultrasound (CDUS), computed tomography angiography (CTA), and magnetic resonance angiography (MRA)] were therefore compared in this study for the relative accuracy in the SCIP flap planning.

Patients and Methods: This study was conducted on eight patients who underwent CDUS, CTA and MRA [3-dimensional time-offlight magnetic resonance angiography, (3D TOF-MRA)] and received reconstructions with the SCIP flaps for head and neck defects. The perforators' locations, courses and calibers were measured or marked for each flap. These imaging preoperative measurements were later compared with intraoperative findings.

Results: CDUS, CTA and 3D TOF-MRA were able to effectively identify the courses of the perforators. 3D TOF-MRA was more accurate at measuring the pedicle calibers of SCIP flaps in comparison with CTA and CDUS.

Conclusion: Three D-TOF-MRA may be a more valuable imaging modality for the preoperative assessment of the vascular anatomy of SCIP flaps.

Keywords: Superficial Circumflex Iliac Artery Perforator Flap, Color Doppler Ultrasound, Computer Tomography Angiography, Magnetic Resonance Angiography, Preoperative Evaluation
\end{abstract}

\section{Background}

The superficial circumflex iliac artery perforator(SCIP) flap has become increasingly popular for reconstruction of various soft tissue defects since its first report by Koshima et al. (1). The versatility of this flap has made it an option for reconstruction of defects in the extremities, trunk, and recently in the head and neck region as well (2-6). SCIP flap presents many advantages, such as less donor-site morbidity, more compatible donor-recipient volume, easy harvesting technique and an inconspicuous donor-site scar (7, 8). However, despite these advantages, SCIP flap may also present some challenges to reconstructive surgeons due to variable locations of perforators, inconstant courses of the pedicle and varying pedicle calibers, thereby increasing the likelihood of reconstructive failure $(4,7,9,10)$.

In order to overcome these problems and to popularize this flap clinically, the anatomic features of each SCIP flap should be assessed preoperatively. Many imaging techniques have thus been attempted in order to facilitate better preoperative evaluation. Color Doppler ultrasound (CDUS) is one of the most commonly used preoperative examinations for many different kinds of perforatorbased flaps (11-13). Computed tomography angiography (CTA) is another choice within the readily available imaging modalities for delineation of the vascular variations of flaps. It could provide us with lots of information regarding the locations, diameters and lengths of the pedicle 
vessels. CTA is now regarded as the gold standard for preoperative vascular mapping, nevertheless its use is always concerned with the risks of radiation exposure and possible allergic reactions to the intravenous administered contrast medium $(13,14)$. In addition to these two methods, magnetic resonance angiography (MRA) also has a role to play in preoperative vascular mapping $(15,16)$. With recent technological developments, a non-invasive, non-contrast, three-dimensional time-of-flight magnetic resonance angiography (3D TOF-MRA) technique was used to acquire data regarding SCIP flap.

Since there is currently no consensus or comparison on using which of the three imaging methods for the preoperative assessment of the perforators and pedicles of SCIP flaps. Thus, all three imaging methods were used in this series of patients for both clinical and research purposes.

\section{Objectives}

The aim of this study was to assess the roles of CDUS, CTA, and 3D TOF-MRA in the preoperative evaluation of the vascular mapping of SCIP flap. CDUS, CTA, and 3D TOF-MRA results were analyzed and compared with direct intraoperative findings, with a hope to tentatively find the most accurate and efficient preoperative imaging modality.

\section{Patients and Methods}

This retrospective study was conducted on the bilateral groin regions of eight patients who all underwent CDUS, CTA, 3D TOF-MRA preoperatively, and all of them received surgeries with SCIP flap reconstructions. A total number of 16 sides of groin regions were analyzed. The patients were informed accordingly and consented to participate in this study with manual signature. Institutional Clinical Research Supervision and Ethics Committee approved this study. The selection criteria were as follows: (1) age $<65$ years; (2) BMI $\leq 28$; (3) no history of extremity trauma, peripheral vascular diseases or severe diabetes mellitus; (4) no prior history of chemo- or radiotherapy.

All surgical procedures were undertaken at the same institution with the same chief and assisting surgeons. The same radiology team was involved in performing the investigations and the interpretation of imaging findings. All eight patients accepted to undergo CDUS, CTA, and 3D TOFMRA to preoperatively assess the perforator vessels position and caliber. CDUS, CTA, and 3D TOF-MRA results were compared with intraoperative findings. The specific imaging protocols were elaborated.

\subsection{CDUS Protocol}

The ultrasound equipment used was a GE Healthcare Voluson E8 (GE Healthcare, Zipf, Austria) with a 10 to 13 $\mathrm{MHz}$ transducer. Considering the complicated vascular anatomy in groin regions, we needed to distinguish between the ascending branches of the deep circumflex iliac arteries (DCIA) and superficial circumflex iliac arteries (SCIA). The locations of the perforators and the courses of the arteries were then marked directly to the overlying skins. The hemodynamic parameters were also recorded at the same time. (Table 1).

\begin{tabular}{lccc}
\hline Table 1. Hemodynamic Parameters of $\mathrm{CDUS}^{\mathrm{a}}$ & \\
\hline & Caliber, $\mathbf{~ m m}$ & $\begin{array}{c}\text { Systolic peak velocity } \\
\mathbf{~ C m} / \mathbf{s}\end{array}$ & Resistance index \\
\hline $\mathbf{1}$ & 0.9 & 23.8 & 0.75 \\
\hline $\mathbf{2}$ & 1.5 & 31.6 & 0.71 \\
\hline $\mathbf{3}$ & 1.2 & 28.2 & 0.74 \\
$\mathbf{4}$ & 1.0 & 16.7 & 0.82 \\
\hline $\mathbf{5}$ & 1.1 & 30.4 & 0.81 \\
\hline $\mathbf{6}$ & 1.2 & 19.5 & 0.83 \\
\hline $\mathbf{7}$ & 1.1 & 37.9 & 0.76 \\
$\mathbf{8}$ & 1.0 & 19.1 & 0.87 \\
\hline Values & $1.13 \pm 0.18$ & $25.90 \pm 7.35$ & $0.79 \pm 0.05$ \\
\hline
\end{tabular}

Abbreviation: CDUS, color Doppler ultrasonography.

${ }^{\mathrm{a}}$ Values are expressed as mean \pm SD.

\subsection{CTA Protocol}

A SIEMENS SOMATOM Definition Flash 64multidetector-row computed tomography scanner (Siemens Medical Solutions, Forchheim, Germany) was used. Scan parameters are summarized in Table 2. A standard bolus of $100 \mathrm{~mL}$ of intravenous Omnipaque 350 (Yangtze River Pharmaceutical [Group] Co., Ltd., Jiangsu, China) was used as a contrast medium. The images were reformatted into maximum-intensity projection and 3D volume-rendered reconstructions using commercially available software (Philips Medical Systems, Royal Dutch Philips Electronics Ltd., Amsterdam, Netherlands). Anatomical features of the perforators were documented, along with the branching patterns of the SCIAs.

The CT scanning technique used in our study was initially based on a protocol used for CTA of the deep inferior epigastric artery (DIEA) in literature (14), and we thus made minor modifications in order to acquire the best parameters for SCIA. The scanning field ranged approximately $5 \mathrm{~cm}$ above the anterior superior iliac spine (ASIS) in a caudal-to-cranial direction up to a point $5 \mathrm{~cm}$ below the pubic symphysis. After injecting the contrast medium, 


\begin{tabular}{|c|c|}
\hline Scanner & Siemens somatom \\
\hline Type & $\begin{array}{l}\text { Definition Flash } 64 \text { multidetector row } \\
\text { computed tomography scanner }\end{array}$ \\
\hline Slice thickness, mm & 1.0 \\
\hline Detector pitch & 0.8 \\
\hline Tube potential, kV & 120 \\
\hline Tube current, mA/effective $\mathrm{mA}$ & $\begin{array}{l}180-200 \text {, depending on the patient's } \\
\text { girth }\end{array}$ \\
\hline IV contrast & Omnipaque $350 ; 100 \mathrm{~mL}$; IVI $4 \mathrm{~mL} / \mathrm{sec}$ \\
\hline Range & $\begin{array}{l}5 \mathrm{~cm} \text { above the anterior superior iliac } \\
\text { spine to } 5 \mathrm{~cm} \text { below the pubic symphysis }\end{array}$ \\
\hline Bolus tracking & Manual trigger \\
\hline
\end{tabular}

Abbreviations: CTA, computed tomography angiography; IV, intravenous; IVI, intravenous injection.

we monitored the femoral artery for the enhancement up to a 5-second delay until the most medium concentrated in the perforator artery.

Axial images from CTA were processed into maximumintensity projection and reformatted into multiple views (Figure 1), including 3D volume-rendered reconstructions (Figure 2) using commercially available software (Extended BrillianceTM Workspace, Royal Dutch Philips Electronics Ltd., Amsterdam, Netherlands). The locations of all the piercing points were measured, marked and recorded according to their distances from the ASIS, while the depth measurement from the groin skin was acquired for surgical planning. The calibers of SCIA perforators were also obtained from CTA data. The coordinates acquired from the imaging measurements were used to transpose the imaging markings to the groin region with the help of 3D reconstructions. The examination time was approximately 15 minutes from preparation to injections. Additional time was needed for data interpretations and image reconstructions using commercially available software.

\subsection{D TOF-MRA Protocol}

All patients received TOF-MRA with a Philips Ingenia 3.0 T magnetic resonance imaging (MRI) scanner (Philips Healthcare; Best, The Netherlands) using a phased array 16channel body coil. TOF-MRA is a non-invasive, non-contrast angiographic technique, which generates images based on flow-related signals. The exploration-scanning field always included the selected surgical field. The patient's position during the TOF-MRA scanning was the same as that during the operation. Scan parameters are summarized in Table 3. The pre-saturation bands typically used in head and neck imaging were omitted in our studies to allow simultaneous visualization of both arterial and venous blood flows.
The images were acquired in three separate slabs, which were then combined to create $3 \mathrm{D}$ images of the vasculatures. The reconstruction voxel size was $0.39 \times 0.39 \times 0.70$ $\mathrm{mm}$, and the acquired matrix size was $500 \times 667$, creating images with high spatial resolutions. The high contrast resolution between flowing blood and stationary tissue allows the creation of 3D tissue-subtracted images of the vasculature. The scan time was around 10 minutes per patient.

\begin{tabular}{ll}
\hline Table 3. 3D TOF-MRA Scan Parameters & \\
\hline Scanner & Philips healthcare \\
\hline Type & $\begin{array}{l}\text { Ingenia } 3.0 \text { T magnetic resonance } \\
\text { imaging scanner }\end{array}$ \\
\hline Act. TR/TE, ms & $23 / 4.9$ \\
\hline Rel. SNR & 1.00 \\
\hline Acquired matrix size & $500 \times 667$ \\
\hline Acquired voxel MPS, mm & $0.40 / 0.60 / 1.40$ \\
\hline Reconstruction voxel MPS, mm & $0.39 \times 0.39 \times 0.70$ mm \\
\hline Range & $\begin{array}{l}5 \mathrm{~cm} \text { above the anterior superior iliac } \\
\text { spine to } 5 \text { cm below the pubic symphysis }\end{array}$ \\
\hline $\begin{array}{l}\text { Abbreviations: MPS, measurement, } \\
\text { signal-noise ratio; 3D TOF-MRA, time of flight magnetic resonance angiography. }\end{array}$
\end{tabular}

The axial images from MR scanning were also reformatted into multiple views (Figure 3 ) and analyzed in a maximum-intensity projection (Figure 4) using commercially available software (Phillips Extended MR Work Space R2.6.3.1). Locations of piercing points were measured in much the same way as with CTA. All information regarding the course of the superficial circumflex iliac arteries was noted. All measured coordinates of perforator locations were transposed to the groin with the help of 3D reconstructions and compared during surgery as well.

\subsection{Operative Procedures}

During the preoperative examinations, the perforator locations had been marked in the bilateral groin areas. In this study, we used CTA as the imaging modality for marking the perforator locations in bilateral groin areas. Perforators' locations in color Doppler sonography and TOFMRA were also marked. The outline of the free flap was designed with the marks by CTA as the center, while encompassing both the nearby marks identified by color Doppler sonography and TOF-MRA in case of inadvertent injury during harvesting. After first cutaneous incision during the operations, the perforators were firstly identified for the exact locations. The accuracy of these three imaging methods were also compared. The SCIP flaps were therefore outlined according to the defect sizes and shapes. The vessels extended beyond the ASIS in a superolateral direction. The first incision was made through the inferomedial border 


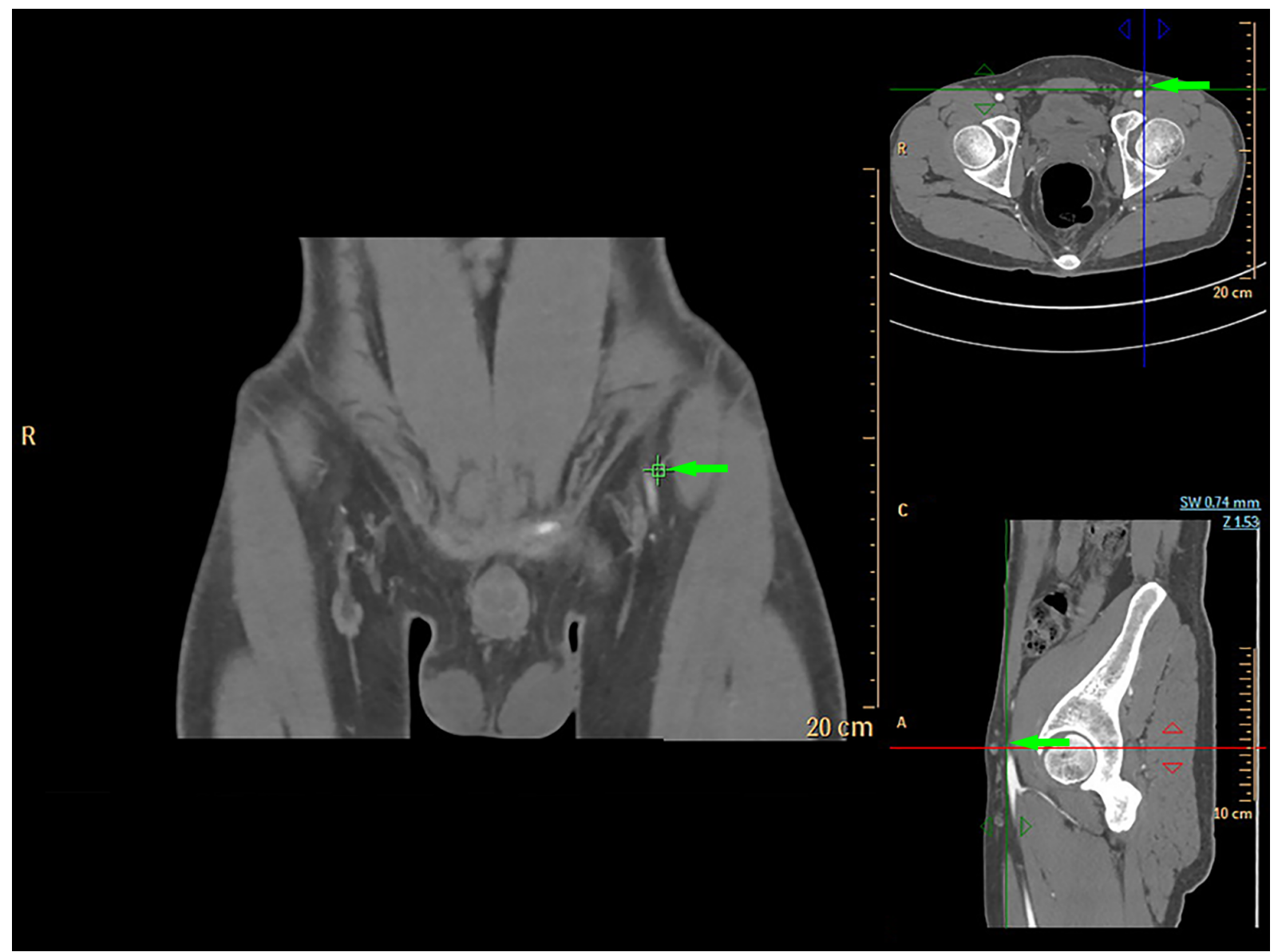

Figure 1. Computed tomography angiography (CTA) multiple views for visualization of the superficial circumflex iliac artery (arrows)

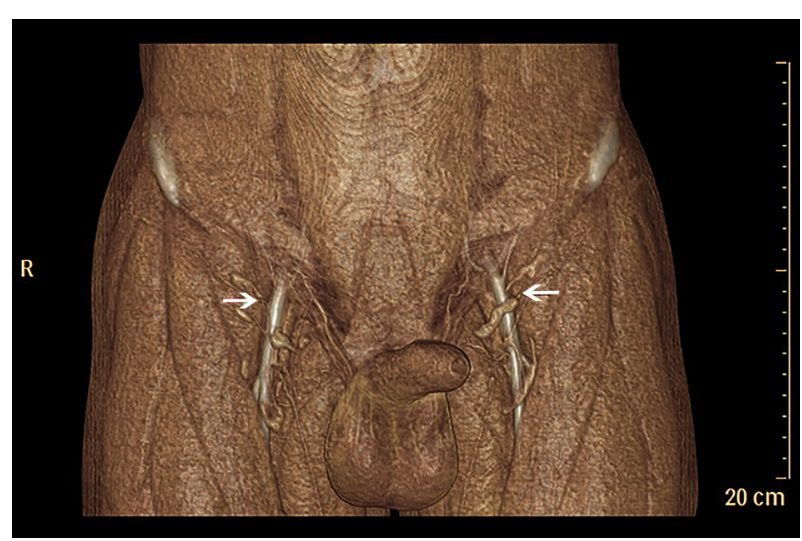

Figure 2. Three-dimensional Computed tomography angiography (CTA) volumerendered reconstructions displaying the superficial circumflex iliac artery (arrows)

of the outlined flaps and the attempts were made to de- tect the exact perforator locations. Afterwards, the piercing points of perforators from the fascia were found with aid of our preoperative markings. The entire courses of the perforators were revealed by suprafascial dissection. All the other branches along the axial vessels were ligated until the main SCIAs were identified (Figure 5).

\subsection{Data Collection}

To be specific, CDUS was examined by two experienced sonographers who frequently cooperated with our surgical team for perforator identification and positioning in lower extremities, such as anterolateral thigh flaps. The characteristics about the perforators of SCIP flaps were confirmed by both sonographers. Besides, CTA was performed by one radiologist and two engineers in the same team. TOF-MRA was performed by another radiologist and two engineers in another team. To increase the reliability and to avoid unnecessary examinations for both imaging visualizations (CTA and TOF-MRA), these imaging meth- 


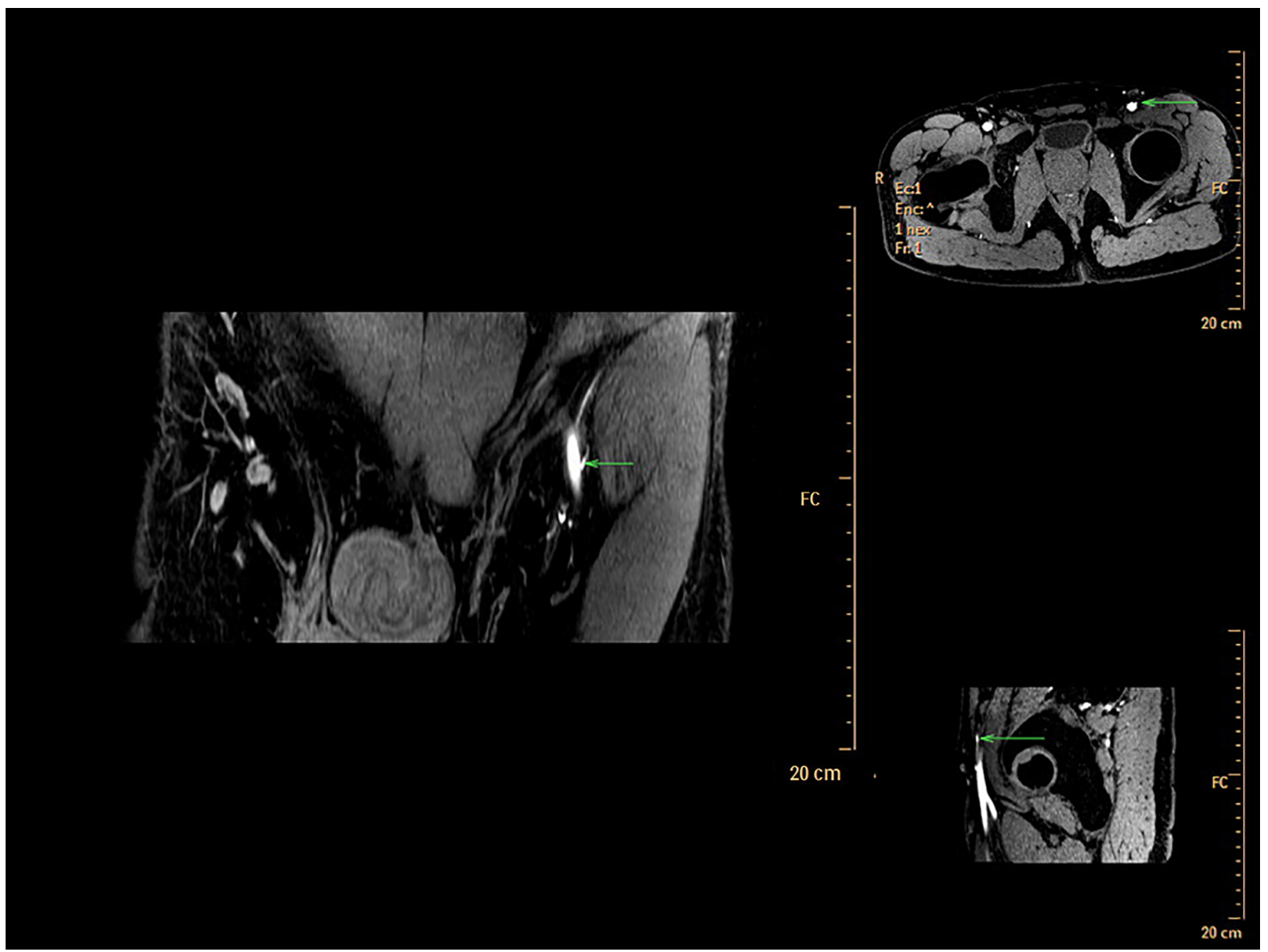

Figure 3. Multiple three dimensional time-of flight magnetic resonance angiography (3D TOF-MRA) displaying the views of the superficial circumflex iliac artery (arrows)

ods were realized with the presence of one surgical team member for confirmation in every single case under the same protocol mentioned in this study. Both CTA and TOFMRA radiologists were unknown (examiner-blind) to the purposes and details of this study. In addition, the imaging examiner (radiologist and technicians) were unaware of the data yielded in other imaging modalities. They perform these different imaging methods individually in an examiner-blind fashion.

\subsection{Statistical Analysis}

The statistical normality was checked by KolmogorovSmirnov test. The paired t-test and repeated measure analysis of variance (ANOVA) were used to compare the data among these imaging groups. Statistical significance was set to $\mathrm{P}$ values lower than 0.05 . All statistical analysis was conducted via SPSS 25 (IBM Corporation, Chicago, Illinois).

\section{Results}

Eight patients underwent all the preoperative imaging examinations including CDUS, CTA, and 3D TOF-MRA. These patients were all successfully reconstructed with the SCIP flaps. Both the preoperative and intraoperative measurements were analyzed. All the variables in this study, specifically, the calibers (diameters), distances (perforators), and depths (perforators) were firstly confirmed for the statistical normality with Kolmogorov-Smirnov test $(\mathrm{P}>0.05)$.

As far as we were concerned, identifying the locations of the perforator's piercing point to the fascia was the crucial step to ensure the safety of raising a SCIP flap (17). The CDUS, CTA, and 3D TOF-MRA were all used in order to mark the piercing points preoperatively and were then compared with our intraoperative findings in order to test the accuracy of each method (Table 4). The calibers of the perforator vessels, crucial for microsurgical anastomosis, was also evaluated and respectively compared with opera- 


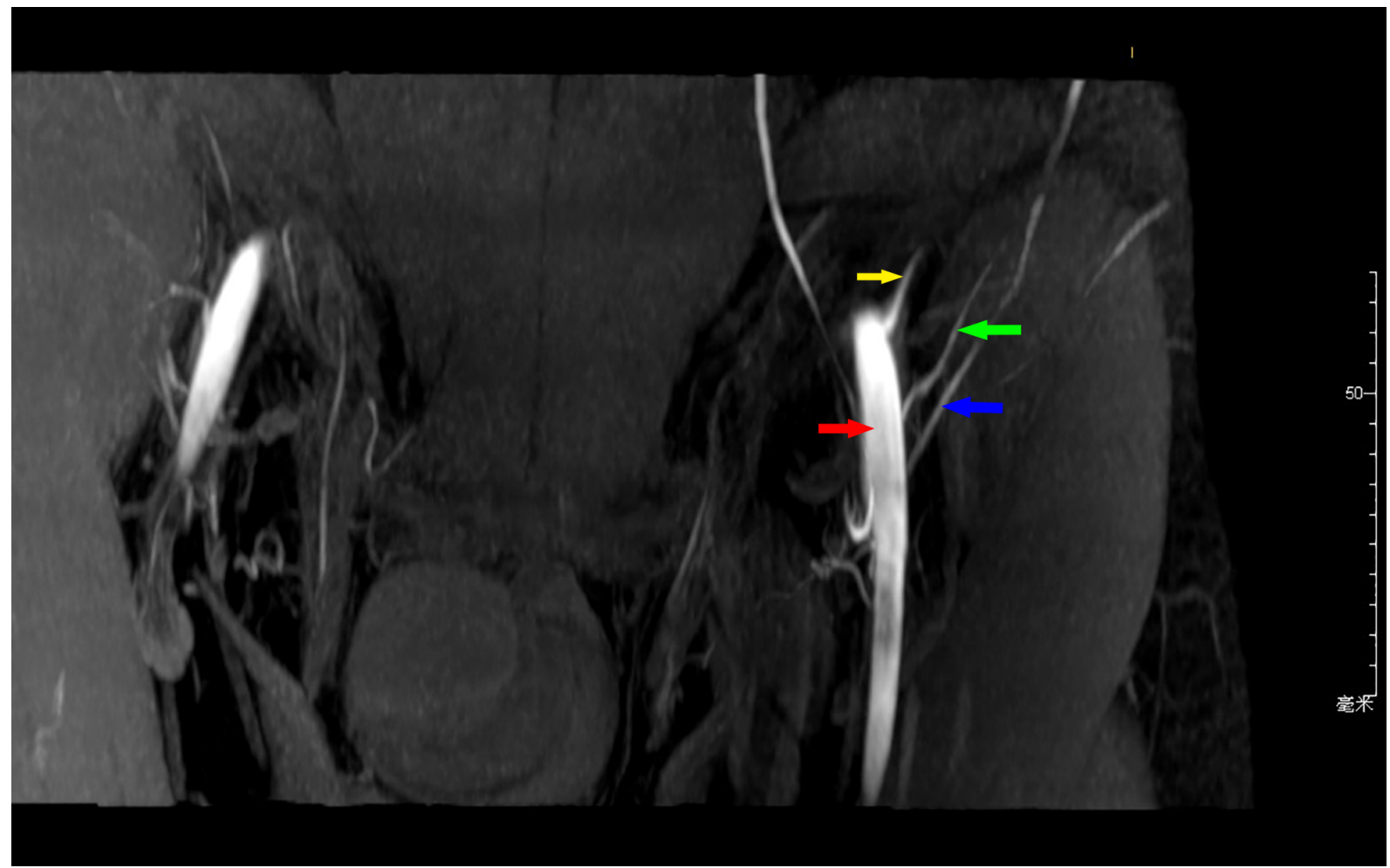

Figure 4. Maximum intensity projection view of the left groin region. Green arrow, superficial circumflex iliac artery; blue arrow, superficial circumflex iliac vein; red arrow, femoral artery; yellow arrow, deep circumflex iliac artery.
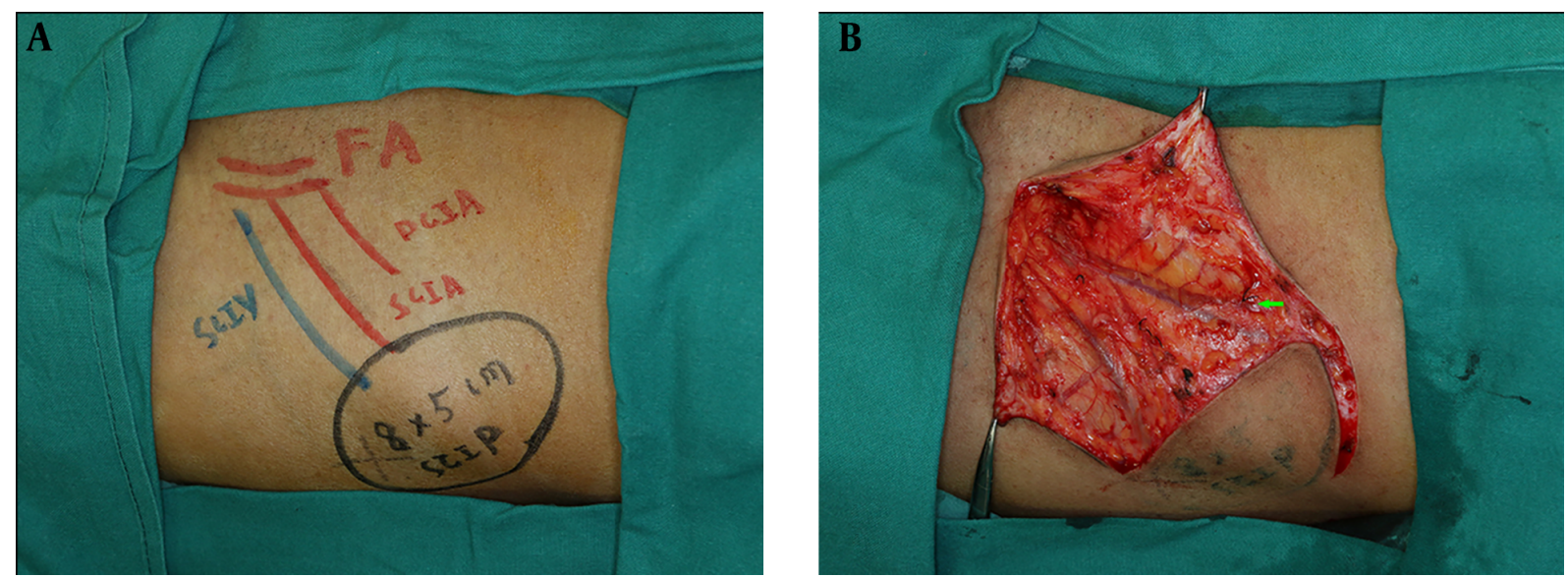

Figure 5. Intraoperative identifications of the perforators (A) and superficial circumflex iliac artery (arrow)(B)

tive findings in the same fashion (Table 5).

In our study, CDUS can assist in marking the course of SCIA, while both CTA and 3D TOF-MRA could clearly display the SCIA systems in the 3D digital reconstructive images. The CTA or MRA images revealed to the surgeons were of three types. The first was a rendered coronal view optimized to highlight the course and insertion points of the SCIA perforators. The second view comprised of axial maximum-intensity projection views demonstrating the starting points of perforators and highlighting the sub- 


\begin{tabular}{|c|c|c|c|c|c|c|c|c|}
\hline & \multicolumn{4}{|c|}{ Distance } & \multicolumn{4}{|c|}{ Depth } \\
\hline & CDUS & CTA & 3D TOF-MRA & OP & CDUS & CTA & 3D TOF-MRA & OP \\
\hline 1 & 4.9 & 5.5 & 5.6 & 5.3 & 1.2 & 1.3 & 1.2 & 1.3 \\
\hline 2 & 6.0 & 6.0 & 5.6 & 6.5 & 1.5 & 1.4 & 1.5 & 1.5 \\
\hline 3 & 6.0 & 5.7 & 5.9 & 5.7 & 1.2 & 1.2 & 1.3 & 1.2 \\
\hline 4 & 5.3 & 5.2 & 5.4 & 4.9 & 1.1 & 1.2 & 1.2 & 1.2 \\
\hline 5 & 4.7 & 3.9 & 4.9 & 4.3 & 1.3 & 1.1 & 1.0 & 1.1 \\
\hline 6 & 5.3 & 5.4 & 4.8 & 5.3 & 0.9 & 0.8 & 0.8 & 0.7 \\
\hline 7 & 4.6 & 5.0 & 5.0 & 5.2 & 0.9 & 0.8 & 0.8 & 0.8 \\
\hline 8 & 4.0 & 4.2 & 4.3 & 3.8 & 1.1 & 1.2 & 1.1 & 1.1 \\
\hline Values & $5.10 \pm 0.69$ & $5.11 \pm 0.73$ & $5.18 \pm 0.53$ & $5.13 \pm 0.83$ & $1.15 \pm 0.20$ & $1.13 \pm 0.22$ & $1.11 \pm 0.24$ & $1.11 \pm 0.26$ \\
\hline
\end{tabular}

Abbreviations: ASIS, anterior superior iliac spine; CDUS, color Doppler ultrasonography; CTA, computed tomography angiography; OP, intraoperative findings; 3D TOFMRA, three dimensional time of flight magnetic resonance angiography.

${ }^{\mathrm{a}}$ Values are ere expressed as mean $\pm \mathrm{SD}$.

\begin{tabular}{|c|c|c|c|c|}
\hline & CDUS & CTA & 3D TOF-MRA & OP \\
\hline $\mathbf{1}$ & 0.9 & 0.8 & 0.7 & 0.5 \\
\hline 2 & 1.5 & 1.3 & 1.2 & 1.2 \\
\hline 3 & 1.2 & 1.1 & 0.9 & 0.8 \\
\hline 5 & 1.1 & 1.0 & 0.9 & 0.6 \\
\hline 6 & 1.2 & 0.9 & 0.8 & 0.7 \\
\hline 7 & 1.1 & 1.0 & 0.8 & 0.8 \\
\hline 8 & 1.0 & 0.9 & 0.9 & 0.7 \\
\hline Values & $1.13 \pm 0.18$ & $0.99 \pm 0.16$ & $0.88 \pm 0.15$ & $0.76 \pm 0.21$ \\
\hline
\end{tabular}

Abbreviations: CDUS, color Doppler ultrasonography; CTA, computed tomography angiography; OP, intraoperative findings; 3D TOF-MRA, three dimensional time of flight magnetic resonance angiography.

${ }^{\mathrm{a}}$ Values are ere expressed as mean $\pm \mathrm{SD}$.

cutaneous and intramuscular courses of the perforators at different anatomical levels. The third was composed of volume-rendered views, optimized in three dimensions to highlight the perforators and their 3D anatomical locations. By using the appropriate software, 3D rotations enabled the selected images to differentially highlight the structures of interest.

CDUS, CTA, and 3D TOF-MRA were all able to effectively identify the positions of the SCIAs. The exact locations of the perforators of the SCIAs during surgery were all in a $0.8 \mathrm{~cm}$ diameter circle area centered on the piercing point marked preoperatively. The distances between the piercing points and anterior superior iliac spines measured by CDUS, CTA and MRA were also compared with the data measured during surgery (Table 4). The repeated measure ANOVA results showed that there was no significant dif- ference between the three groups and intraoperative findings $(P=0.949)$. The sensitivities for identifying the depths of the artery piercing points via CDUS, CTA, 3D TOF MRA and operation were statistically identical $(\mathrm{P}=0.877)$. Compared with the calibers measured during the operations, all these methods (CUDS, CTA, and 3D TOF-MRA) were unable to estimate the intra-operative calibers according to statistical analysis $(\mathrm{P}=0.02)$. However, after comparing the relative data between CDUS, CTA, 3D TOF-MRA, and the intraoperative findings, 3D TOF-MRA was significantly superior at mimicking the exact diameters of the perforator vessels compared to CTA and CDUS (P CDUS vs. OP $=0.020$, mean CDUS-OP $=0.3625$; P CTA vs. OP $=0.015$, mean CTA$\mathrm{OP}=0.2250$; $\mathrm{P}$ TOF-MRA vs. $\mathrm{OP}=0.033$, mean TOF-MRA-OP $=$ $0.1125)$.

Agreements of CDUS, CTA, 3D-TOF MRA and operative 
assessment for measuring distance between the perforator point and ASIS were assessed by Bland-Altman analyses (between all possible pairwise selections of these four measurements). In each of these plots (totally six plots), the data were located between agreement band showing acceptable agreement between these four methods. Similar analyses were performed for depth of the perforators' piercing point and caliber of the perforator vessel that again showed agreement between these four methods [except for CDUS vs. 3D-TOF MRA of depth of perforators piercing point and CDUS vs. CTA of caliber of the perforator vessel; in both of them, only data of one patient were located beyond the agreement band]. This shows an agreement for depth and caliber measurements between these four methods.

\section{Discussion}

Free flaps have been applied in many clinical scenarios for nearly three decades. With advancement in knowledge and techniques, the survival rates of different vascularized flaps were reported as high as 96\% - 98\% (18). Recent focus in this field is shifting to increase flap aesthetics, to improve flap designs and to reduce donor-site morbidities $(19,20)$. Since the introduction of the novel concept of "reconstructive ladders" (19, 21), perforator flaps have been widely acknowledged as the primary reconstructive treatment options as they meet the needs of the recent shifting focus. Among various perforator-based flaps, SCIP flap was recommended as an ideal "like-with-like" tissue option for reconstruction of complex defects at minimal donor-site "cost" and with maximal efficacy. Yet the challenges of harvesting such flaps remained huge, in terms of their variable calibers and locations of the perforators.

Many imaging modalities have now been utilized to assess the donor-site vascularities in the preoperative settings, though no consensus has been reached so far. Generally speaking, CTA, MRA and CDUS are the three commonly used imaging methods in perforator-based reconstructive surgeries. According to studies with regards to perforator flaps, CTA was the most conventionally utilized preoperative method. However, CDUS was also applied to the candidates for perforator-flap reconstructions in some studies. The ideal imaging modality should meet several key criteria. It should give the most accurate information about travelling courses and calibers of the perforating vessels down to the sub-millimeter level. It should also be highly reproducible, and have low false signals compared with intraoperative findings. Besides, the imaging technology should be as inexpensive, and readily available as possible. Radiation exposure was another concern allowing the test to be used in a routine screening capacity (22).
The merits of CDUS are obvious, which include no intravenous contrast, and no radiation exposure. In addition, it is an imaging technique with additional hemodynamic data (blood flow velocities). Tashiro et al. (23) have already proved this in their study, which was consistent with our results. However, CDUS has many disadvantages. It is relatively inaccurate in caliber detecting and it is a technician-dependent procedure and non-reproducible. Evidence on applying CDUS alone in the perforator-based flaps evaluation, especially in the abdominal regions, is not compelling due to the inconsistent data from a lot of studies. Blondeel et al. (24) and Giunta et al. (11) have reported a very high proportion of false positive results. From their study, ultrasonography did not seem to be the best option.

In 2012, Pratt presented an objective evidence-based review of the literature about preoperative imaging of perforator vasculatures in planning microvascular reconstructions (13). They summarized that sufficient evidence existed in demonstrating that CTA was the gold standard for perforator mappings. Several other studies also suggested that the perforator-based flap designs via CTA reduced the risks of flap complications and associated donor site morbidity for breast reconstructions $(13,25,26)$. In our study, CTA identified SCIAs in 93.8 percent (15/16) of the total 16 sides. The location of the perforators identified by CTA was found in a $0.8 \mathrm{~cm}$ diameter circle area. This was later verified during harvesting of the flap intraoperatively. CTA was significantly sensitive in identifying SCIA perforators preoperatively. Comparing the results between CTA and intraoperative findings, CTA was also able to effectively evaluate the calibers of the perforator vessels. As was shown in our results, the branching patterns were optimally displayed using 3D reconstruction maximum-intensity projection images.

A few studies assessing the use of MRA for preoperative detections of perforator arteries can be found in the literature $(16,27)$. The use of MRA seems to be associated with the concern of being an expensive and time-consuming modality. However, MRA has its own advantages over the previously described two techniques. It minimizes radiation exposure and has a better muscle-to-vessel contrast. The use of contrast-enhanced MRA has been previously described for preoperative SCIP flap designs. According to these studies, it has the advantages of a cross-sectional imaging technique, providing accessible images to the surgeons, while not exposing the patient to ionizing radiation. In order to avoid the injection of contrast agents for MRA images, we came up with a novel 3D TOF-MRA technique for perforator-based flap designs. This unique non-invasive imaging modality provides actionable images without contrast or radiation exposure and can provide an anatomic map with the same accuracy. The results 
of our study also revealed that 3D TOF-MRA was able to correctly identify and measure the lengths of SCIA perforators, and to measure the caliber in much the same way as other invasive MRA techniques. Meanwhile, the examination via 3D TOF-MRA is cheaper than CTA according to our results.

Both 3D TOF-MRA and CTA have been proven to be highly effective and accurate at imaging perforators for the SCIA perforator flap harvest. However, they required a significant time investment for imaging evaluations on the part of both radiologists and operating surgeons, and additional costs were involved in the use of these modalities. Both CTA and 3D TOF-MRA were very accurate for localizing the course of the perforator vessels, and the images can be visually and repetitively analyzed preoperatively. From our perspective, 3D TOF-MRA is better than CTA because patients can avoid ionizing radiation and contrast agents. In addition, 3D TOF-MRA was capable of delivering higher resolution scans with reduced scanning times. For these reasons, 3D TOF-MRA presents a very promising advancement in the preoperative evaluation of SCIP flaps.

We were aware of the limitations of our study that the design was retrospective, the sample size was small and the artificial errors could not be avoided after dissecting and ligating the vessels. Admittedly, it is possible that intraoperative measurements may be slightly changed or altered due to surgical dissection and manipulation. Besides, there may also be minor differences between preoperative and intraoperative setting in terms of the vessel calibers, perforator positions and depths of perforators' piercing points. According to other studies with regards to preoperative radiological estimation and intraoperative realization, these "virtual to real" discrepancies in term of different settings were generally unmentioned or neglected. We acknowledged that the predictive power of these preoperative measurements may be slightly affected due to intraoperative manipulations, including dissections and ligations. For the sake of accuracy, we acknowledged these discrepancies. The power analysis was not performed in this study. Thus, we expect to use the implications or preliminary conclusions for a design of future prospective study.

In conclusion, based on our study, 3D TOF-MRA showed the relative superiority in preoperative SCIP flap designs when compared with CDUS and CTA. It was non-invasive, non-irradiated, reproducible, and effective. 3D TOF-MRA might be a promising candidate for the design of flaps derived from groin vascular anatomy.

\section{Footnotes}

Authors' Contribution: Study concept and design: YH and CM. Analysis and interpretation of data: ZT and SW.
Drafting of the manuscript: ZT. Critical revision of the manuscript for important intellectual content: YH and CM. Statistical analysis: ZT and SW.

\section{Conflict of Interests: None.}

Ethical Approval: The patients were informed accordingly and consented to participate in this study with manual signature. Institutional Clinical Research Supervision and Ethics Committee approved this study (code: 2014106).

Funding/Support: Supported by Science and Technology Commission of Shanghai Municipality, Science and Technology Innovation Action Plan, grant number: 17511110300; Natural Science Foundation, grant number: 19ZR1430000; Shanghai Municipal Health Commission Foundation, grant number: 20194 Y0017.

Informed Consent: Informed Consent were well explained and signed by all the patients.

\section{References}

1. Koshima I, Nanba Y, Tsutsui T, Takahashi Y, Urushibara K, Inagawa $\mathrm{K}$, et al. Superficial circumflex iliac artery perforator flap for reconstruction of limb defects. Plast Reconstr Surg. 2004;113(1):233-40. doi: 10.1097/01.PRS.0000095948.03605.20. [PubMed:14707641].

2. Koshima I, Nanba Y, Nagai A, Nakatsuka M, Sato T, Kuroda S. Penile reconstruction with bilateral superficial circumflex iliac artery perforator (SCIP) flaps. J Reconstr Microsurg. 2006;22(3):137-42. doi: 10.1055/s2006-939957. [PubMed: 16780040].

3. Hong JP, Sun SH, Ben-Nakhi M. Modified superficial circumflex iliac artery perforator flap and supermicrosurgery technique for lower extremity reconstruction: A new approach for moderate-sized defects. Ann Plast Surg. 2013;71(4):380-3. doi: 10.1097/SAP.0b013e3182503ac5. [PubMed: 23187712].

4. Kim JH, Kim KN, Yoon CS. Reconstruction of moderate-sized distal limb defects using a superthin superficial circumflex iliac artery perforator flap.J Reconstr Microsurg. 2015;31(9):631-5. doi: 10.1055/s-00351558959. [PubMed: 26220431].

5. Nasir S, Aydin MA. Versatility of free SCIA/SIEA flaps in head and neck defects. Ann Plast Surg. 2010;65(1):32-7. doi: 10.1097/SAP.0b013e3181bb4b24. [PubMed: 20574218].

6. Green R, Rahman KM, Owen S, Paleri V, Adams J, Ahmed OA, et al. The superficial circumflex iliac artery perforator flap in intra-oral reconstruction. J Plast Reconstr Aesthet Surg. 2013;66(12):1683-7. doi: 10.1016/j.bjps.2013.07.011. [PubMed: 23982067].

7. Strobbe S, Van Landuyt K, Delaere P, Vander Poorten V, Vanclooster C. Superficial circumflex iliac artery perforator flap for reconstruction of oral defects after tumor resection. B-ENT.2015;11(2):157-61. [PubMed: 26563018].

8. Iida T, Mihara M, Yoshimatsu H, Narushima M, Koshima I. Versatility of the superficial circumflex iliac artery perforator flap in head and neck reconstruction. Ann Plast Surg. 2014;72(3):332-6. doi: 10.1097/SAP.ob013e318260a3ad. [PubMed: 22868321].

9. He Y, Tian Z, Ma C, Zhang C. Superficial circumflex iliac artery perforator flap: Identification of the perforator by computed tomography angiography and reconstruction of a complex lower lip defect. Int J Oral Maxillofac Surg. 2015;44(4):419-23. doi: 10.1016/j.ijom.2014.11.001. [PubMed: 25487563].

10. Ma C, Tian Z, Kalfarentzos E, He Y. Superficial circumflex iliac artery perforator flap: A promising candidate for large soft tis- 
sue reconstruction of retromolar and lateral buccal defects after oncologic surgery. J Oral Maxillofac Surg. 2015;73(8):1641-50. doi: 10.1016/j.joms.2014.12.022. [PubMed: 25979557].

11. Giunta RE, Geisweid A, Feller AM. The value of preoperative Doppler sonography for planning free perforator flaps. Plast Reconstr Surg. 2000;105(7):2381-6. doi: 10.1097/00006534-200006000-00011. [PubMed: 10845290].

12. Hallock GG. Doppler sonography and color duplex imaging for planning a perforator flap. Clin Plast Surg. 2003;30(3):347-57. v-vi. doi: 10.1016/s0094-1298(03)00036-1. [PubMed: 12916592].

13. Pratt GF, Rozen WM, Chubb D, Ashton MW, Alonso-Burgos A, Whitaker IS. Preoperative imaging for perforator flaps in reconstructive surgery: A systematic review of the evidence for current techniques. Ann Plast Surg. 2012;69(1):3-9. doi: 10.1097/SPA.0b013e318222b7b7. [PubMed: 22627495].

14. Rozen WM, Phillips TJ, Ashton MW, Stella DL, Gibson RN, Taylor GI. Preoperative imaging for DIEA perforator flaps: A comparative study of computed tomographic angiography and Doppler ultrasound. Plast Reconstr Surg. 2008;121(1):9-16. doi: 10.1097/01.prs.0000293874.71269.c9. [PubMed: 18176200].

15. Neil-Dwyer JG, Ludman CN, Schaverien M, McCulley SJ, Perks AG. Magnetic resonance angiography in preoperative planning of deep inferior epigastric artery perforator flaps. J Plast Reconstr Aesthet Surg. 2009;62(12):1661-5. doi: 10.1016/j.bjps.2008.06.048. [PubMed: 18993122].

16. Rozen WM, Stella DL, Bowden J, Taylor GI, Ashton MW. Advances in the pre-operative planning of deep inferior epigastric artery perforator flaps: Magnetic resonance angiography. Microsurgery. 2009;29(2):11923. doi: 10.1002/micr.20590. [PubMed: 19021232].

17. Hsu WM, Chao WN, Yang C, Fang CL, Huang KF, Lin YS, et al. Evolution of the free groin flap: The superficial circumflex iliac artery perforator flap. Plast Reconstr Surg. 2007;119(5):1491-8. doi: 10.1097/01.prs.0000256057.42415.73. [PubMed: 17415243].

18. Wong AK, Joanna Nguyen T, Peric M, Shahabi A, Vidar EN, Hwang BH, et al. Analysis of risk factors associated with microvascular free flap failure using a multi-institutional database. Microsurgery. 2015;35(1):6-12. doi:10.1002/micr.22223. [PubMed: 24431159].

19. Geddes CR, Morris SF, Neligan PC. Perforator flaps: Evolution, clas- sification, and applications. Ann Plast Surg. 2003;50(1):90-9. doi: 10.1097/00000637-200301000-00016. [PubMed: 12545116].

20. Saint-Cyr M, Schaverien MV, Rohrich RJ. Perforator flaps: History, controversies, physiology, anatomy, and use in reconstruction. Plast Reconstr Surg. 2009;123(4):132-45. doi: 10.1097/PRS.0b013e31819f2c6a. [PubMed: 19337067].

21. Wei FC, Jain V, Suominen S, Chen HC. Confusion among perforator flaps: What is a true perforator flap? Plast Reconstr Surg. 2001;107(3):874-6. doi: 10.1097/00006534-200103000-00037. [PubMed: 11304620].

22. Rozen WM, Garcia-Tutor E, Alonso-Burgos A, Acosta R, Stillaert F, Zubieta JL, et al. Planning and optimising DIEP flaps with virtual surgery: The Navarra experience. J Plast Reconstr Aesthet Surg. 2010;63(2):28997. doi:10.1016/j.bjps.2008.10.007. [PubMed: 19042174].

23. Tashiro K, Harima M, Kato M, Yamamoto T, Yamashita S, Narushima $\mathrm{M}$, et al. Preoperative color Doppler ultrasound assessment in planning of SCIP flaps. J Plast Reconstr Aesthet Surg. 2015;68(7):979-83. doi: 10.1016/j.bjps.2015.03.004. [PubMed: 25824198].

24. Blondeel PN, Beyens G, Verhaeghe R, Van Landuyt K, Tonnard P, Monstrey SJ, et al. Doppler flowmetry in the planning of perforator flaps. Br J Plast Surg. 1998;51(3):202-9. doi: 10.1016/s0007-1226(98)80010-6. [PubMed: 9664879].

25. Teunis T, Heerma van Voss MR, Kon M, van Maurik JF. CTangiography prior to DIEP flap breast reconstruction: A systematic review and meta-analysis. Microsurgery. 2013;33(6):496-502. doi 10.1002/micr.22119. [PubMed: 23836386].

26. Casey W3, Chew RT, Rebecca AM, Smith AA, Collins JM, Pockaj BA Advantages of preoperative computed tomography in deep inferior epigastric artery perforator flap breast reconstruction. Plast Reconstr Surg. 2009;123(4):1148-55. doi: 10.1097/PRS.ob013e31819e23e1. [PubMed: 19337083].

27. Alonso-Burgos A, Garcia-Tutor E, Bastarrika G, Benito A, Dominguez PD, Zubieta JL. Preoperative planning of DIEP and SGAP flaps: Preliminary experience with magnetic resonance angiography using 3-tesla equipment and blood-pool contrast medium. J Plast Reconstr Aesthet Surg. 2010;63(2):298-304. doi: 10.1016/j.bjps.2008.11.009. [PubMed: 19121986]. 